abgefasst worden war. Deshalb wird dort zunächst auch nur die Summe ausbezahlt, die durch die Bereinigung mit der jeweiligen KV (Regelleistungen plus extrabudgetäre Leistungen) abgedeckt ist. Dazu kommt als Aufschlag eine Pauschale von $10 \%$ der Bereinigung als reine Vorfinanzierung. Die Ermittlung der endgültigen Vergütung erfolgt, wenn der tatsächliche Umfang der kompensatorische Einsparungen und Effizienzsteigerungen fixierbar ist. Start ist der 1. April 2012, die erste Effektivitätskontrolle findet dann Anfang 2015 statt.

\section{MMW Kommentar}

Der Grundsatz der "Bierdeckel-Abrechnung", wie sie seit nun schon drei Jahren in BadenWürttemberg erfolgreich praktiziert wird, musste damit (zunächst) verlassen werden. Umso mehr gilt es, dass die Forderung, den $\S 73 b$ neu politisch wieder durch den $\$ 73 b$ alt (hier ist die Refinanzierung über KV-Niveau keine Pflicht) zu ersetzen, weiter konsequent verfolgt werden muss. Immerhin ist dieser Schritt bereits Gegenstand des Parteiprogramms der SPD für die nächste Bundestagswahl. Für eine Übergangszeit können aber auch solche Verträge der dringenden Notwendigkeit einer Vertragsautonomie für die Hausärzte gerecht werden.

Das nicht mehr nachvollziehbare Vergütungssystem im Kollektivvertrag mit einer immer strengeren Morbiditätsanbindung wird der Arbeit und dem Aufgabengebiet der Hausärzte nicht gerecht. Nicht zuletzt deshalb wählen immer weniger Studienabgänger den Weg in die hausärztliche Versor- gung. Eine Morbiditätsentwicklung als Grundlage des Honorarvolumens führt für die Hausärzte in eine gefährliche Sackgasse.

Schließlich ist es die Aufgabe des Hausarztes, durch präventive Maßnahmen - im Zusammenwirken mit Fachspezialisten - Morbidität zu verhindern oder zumindest deren (finanzielle) Auswirkungen abzuschwächen. Diesem Leistungsspektrum werden nur die Hausarztverträge nach §73b SGB $\checkmark$ gerecht. Was im Moment noch fehlt, aber-insbesondere bei einem Regierungswechsel - durchaus noch erreicht werden kann, ist die korrekte finanzielle Ausstattung solcher Hausarztverträge, wie in Baden-Württemberg vorbildlich praktiziert.

\title{
Bringt das neue Pflegegesetz auch ein angemessenes Betreuungshonorar?
}

- Der aktuelle Referentenentwurf für ein Pflege-Neuausrichtungsgesetz (PNG) beinhaltet, dass die Kassenärztlichen Vereinigungen in §119b Abs. 2 SGB V künftig verpflichtet werden sollen, auf Antrag der Pflegeeinrichtung Kooperationsverträge zwischen Pflegeeinrichtungen und Leistungserbringern „zu vermitteln“. Näheres zu dieser Verpflichtung bzw. Sanktionen bei Nichterfüllung dieser Verpflichtung wird nicht geregelt.

Zudem werden die Partner der Bundesmantelverträge - KVen und Kassen - beauftragt, die Anforderungen an eine kooperative und koordinierte ärztliche und pflegerische Versorgung von Pflegebedürftigen in stationären Pflegeeinrichtungen zu vereinbaren. Als Frist wird ein Jahr nach Inkrafttreten festgelegt. Hinzu kommt die Möglichkeit, in §87a Abs. 2 Satz 3 SGB V Zuschläge auf den Orientierungswert für besonders förderungswürdige
Leistungen bzw. für Leistungen besonders förderungswürdiger Leistungserbringer in unterversorgten Gebieten vereinbaren zu können. Diese wird erweitert um die Möglichkeit, solche Zuschläge auch zur Förderung der koordinierten kooperativen ärztlichen und pflegerischen Versorgung von Pflegebedürftigen in stationären Pflegeeinrichtungen oder von Kooperationsverträgen vereinbaren zu können. Die Bundesregierung kalkuliert dabei einen finanziellen Mehrbedarf von 77 Millionen Euro für diese Zuschläge.

\section{MMW Kommentar}

Das Gesetz würde - kommt es so, wie jetzt vorgeschlagen - eine große Lücke in der ambulanten, insbesondere hausärztlichen Versorgung schließen. Die besondere Betreuung von Patienten in Pflegeheimen ist nämlich nicht in der Gebührenordnung (EBM) abgebildet. Die notwendigen Leistungen erschleichen sich die Kassen regelrecht, indem sie so tun, als könne das über die Leistungspositionen für Patienten, die nicht in solchen Einrichtungen untergebracht sind, erledigt werden. Die Folge ist eine immer größere Versorgungs/ücke, die nur punktuell und regional durch Sonderverträge geschlossen wird.

Als vorbildlich können hier die „Pflegeheimverträge" in Bayern angesehen werden. Was noch fehlt, ist allerdings eine Verpflichtung der gesetzlichen Kassen, solche Leistungen zu finanzieren, wie dies für die hausarztzentrierte Versorgung im alten $\$ 73 b$ SGB $\checkmark$ geregelt war. Denn freiwillig werden die "Sparkassen" weder Verträge abschließen noch den Pflegeheimen die notwendige finanzielle Ausstattung verschaffen, um die ärztlichen Leistungen aus solchen Verträgen zu finanzieren. 